\title{
The effect of age and experience on inhibition of the acoustic startle response by gaps in background noise
}

\author{
KAREN F. DEAN \\ U.S. Environmental Protection Agency, Research Triangle Park, North Carolina \\ LARRY P. SHEETS \\ Mobay Corporation, Stilwell, Kansas \\ and \\ KEVIN M. CROFTON and LAWRENCE W. REITER \\ U.S. Environmental Protection Agency, Research Triangle Park, North Carolina
}

\begin{abstract}
The acoustic startle response (ASR) is inhibited when the eliciting stimulus is preceded by a brief gap in background noise. The present study examined the ontogeny of ASR gap inhibition in the rat and the role of experience on its development. Independent groups of Long-Evans rats were tested for 3 consecutive days, at one of eight ages ranging from Postnatal Day (PND) 14-16 to PND 98-100. Gaps $(0,2,4,8,16,32$, or $64 \mathrm{msec})$ in broad-band background noise $(80 \mathrm{~dB}$ on $/ 35 \mathrm{~dB}$ off) were presented $190 \mathrm{msec}$ prior to the eliciting stimulus (a 13-kHz, 120-dB, 40-msec tone with 2.5-msec rise/decay). The results show that longer gaps (16-64 msec) inhibited the ASR at all ages; however, the magnitude of inhibition increased with age and repeated testing. The efficacy of shorter gaps (4-8 msec) in the inhibition of the ASR improved until PND 35-37, when the adult pattern was achieved. These findings indicate that both the magnitude of ASR gap inhibition and the efficacy of gaps of less than $16 \mathrm{msec}$ are dependent on the age and experience of the animal.
\end{abstract}

The acoustic startle response (ASR) in rats can be modified by sensory events that precede the eliciting stimulus (see Davis, 1984; Hoffman \& Ison, 1980, for reviews of reflex modification). Auditory prestimuli that have been shown to modify the ASR include steady levels of background noise, which sensitize the response, and a brief gap in ongoing background noise, or a pulse of lowintensity noise, which inhibit the reflex (Hoffman \& Fleshler, 1963; Hoffman \& Searle, 1965; Ison, 1982). Reflex modification of the ASR has been shown to provide a sensitive and reliable measure of sensory function (see Ison, 1988, for review). Inhibition of the ASR by tone pulses provides an objective measure of stimulus detection

Portions of this manuscript were presented as a poster at the 18 th Annual Meeting of the Society for Neuroscience, November 15, 1988, Toronto, Canada (Society for Neuroscience Abstracts, 4, 425). The research described in this article has been reviewed by the Health Effects Research Laboratory, U.S. Environmental Protection Agency, and approved for publication. Approval does not signify that the contents necessarily reflect the views and policies of the Agency, nor does mention of trade names or commercial products constitute endorsement or recommendation for use. Address correspondence to Kevin M. Crofton, Neurotoxicology Division (MD-74B), Health Effects Research Laboratory, U.S. Environmental Protection Agency, Research Triangle Park, NC 27711.

-Accepted by previous editor, Lynn Nadel
(Young \& Fechter, 1983), and inhibition by gaps has been identified with temporal acuity (Ison, 1982; Kellogg, Ison, \& Miller, 1983).

The present study focuses on the ontogeny of gap detection in rats. In humans, this function develops relatively slowly over a period of 6-8 years (Davis \& McCroskey, 1980 ) and is considerably retarded in comparison with threshold detection for simple tones. In rats, reflex modification of the ASR using background noise and auditory prepulse has been reported to occur between Postnatal Day (PND) 12 and PND 16, shortly after the ASR is first elicited (Kellogg, Tervo, Ison, \& Parisi, 1980; Parisi \& Ison, 1979, 1981; Sheets, Dean, \& Reiter, 1988). Following the initial occurrence of reflex modification, the developmental profile continues to change until the adult pattern emerges around PND 16-21 (Kellogg et al., 1980; Parisi \& Ison, 1979; Sheets et al., 1988). Parisi and Ison (1979) have noted that the development of these mature patterns coincides with the maturation of auditory acuity, as measured by cochlear microphonics (Crowley \& HeppRaymond, 1966). Although there has been no study specifically designed to define the ontogeny of gap inhibition in rats, it has been shown to develop between PND 25 and PND 28 (Kellogg et al., 1983). However, the primary concern of that study was to examine the effects of prenatal diazepam and not to define the ontogeny of gap inhibition per se. Consequently, critical parameters such as the 
initial age of testing, the time interval between test sessions, and the effect of test experience were not examined.

In studies designed specifically to define the ontogeny of other measures of reflex modification, such as sensitization and prepulse inhibition, testing began between PND 10 and PND 13 and continued at close intervals (daily or every 2 days) through PND 21 (Kellogg et al., 1980; Parisi \& Ison, 1979, 1981; Ruppert, Dean, \& Reiter, 1983; Sheets et al., 1988). In addition, because repeated testing of animals is routine in ontogeny studies, experiential effects have been examined (Parisi \& Ison, 1979; Ruppert et al., 1983; Sheets et al., 1988). Although no differences between naive and experienced rats have been found for sensitization or prepulse inhibition of the ASR, preliminary evidence from our laboratory indicates that ASR gap inhibition shows considerable change over the course of the first three test sessions, for both weanling and adult rats (Crofton, Sheets, Dean, \& Peele, 1988; Dean, Sheets, Crofton, \& Reiter, 1988). Our observations prompted us to question the role of repeated testing in the previous report of gap ontogeny (Kellogg et al., 1983), in which significant gap inhibition was not found until the third test experience, on PND 28. The initial age of testing, the spacing of test intervals, and the experience of repeated testing could be critical factors in determining the ontogeny of a response. Furthermore, to make equitable comparisons between the ontogeny of various responses, these factors should be closely matched.

The purpose of the present experiment, therefore, was to describe the ontogeny of auditory gap inhibition of the ASR in naive rats and to determine the effect of test experience over three daily sessions.

\section{METHOD}

\section{Animals}

Pregnant Long-Evans hooded rats (Charles River, Inc.) were received on Day 15 of gestation. The animals were housed individually in suspended plastic cages with food (Purina Lab Chow) and water available ad lib. The rats were kept in a facility that was maintained on a 12:12 h light:dark photoperiod (lights on 0600-1800 h), with controlled temperature $\left(22^{\circ} \pm 2^{\circ} \mathrm{C}\right)$ and relative humidity $(40 \% \pm 20 \%)$. On PND 1 (day of birth = PND 0), pups from all litters were randomized and redistributed, with each dam assigned 4 males and 4 females. One paw of each pup was tattooed for withinlitter identification (modified from Avery \& Spyker, 1977). On PND 23, all pups were weaned and housed by sex with littermates in groups of 4 . All behavioral testing was performed between 0800 and $1600 \mathrm{~h}$.

\section{Apparatus}

Testing was conducted in eight sound-attenuated chambers $(30.5 \times 57 \times 35 \mathrm{~cm})$, each containing a wire-mesh plastic-framed test cage, mounted on a load cell/force transducer assembly (Gould Statham Model UC3 with 5-lb adaptor Model UL4-5) designed to measure vertical force. Test chambers of three different sizes were used according to the age of the rats: PNDs 14-31 $(9 \times 5.1 \times$ $5.1 \mathrm{~cm})$, PNDs 35-51 (12.7 $\times 6.4 \times 6.4 \mathrm{~cm})$, and PNDs 63-100 $(20 \times 7.6 \times 7.6 \mathrm{~cm})$. Two speakers were suspended from the ceiling of each chamber. One speaker (Motorola $5.0 \times 12.5 \mathrm{~cm}$ piezoelectric tweeter) presented the eliciting stimulus (a $13-\mathrm{kHz}$,
120-dB, 40-msec tone with a 2.5-msec rise/decay). A second speaker (Creative Acoustics, $16 \Omega$ ) delivered broad-spectrum background noise at $80 \mathrm{~dB}$. The output from this speaker, measured at $80 \mathrm{~dB}$ with a Spectrascope Model SD330 Real Time Analyzer, had approximately equal power from $200 \mathrm{~Hz}$ to $15 \mathrm{kHz}( \pm 10 \mathrm{~dB})$; output dropped $40 \mathrm{~dB}$ from $15 \mathrm{kHz}$ to $20 \mathrm{kHz}$. All sound measurements were made with a Brüel \& Kjaer Model 2209 Sound Level Meter (A scale) equipped with a Model 41650.5 -in. free-field microphone.

\section{Procedure}

Following a 10-min adaptation period at a low background noise level (approximately $35 \mathrm{~dB}$ ), the animals received a total of 105 trials. Each trial began with $80 \mathrm{~dB}$ of white noise, which continued until $190 \mathrm{msec}$ prior to the presentation of the eliciting stimulus. At this time, the background noise was reduced to $35 \mathrm{~dB}$ for 0 , $2,4,8,16,32$, or $64 \mathrm{msec}$. The background noise resumed prior to the presentation of the eliciting stimulus, thereby producing a brief gap in the background noise. The interstimulus interval (ISI) was fixed at $190 \mathrm{msec}$ from gap onset to the onset of the eliciting stimulus. The ITI (time between the presentation of each eliciting stimulus) was fixed at $15 \mathrm{sec}$. Each rat received 15 trials at each of the seven gap durations. The order of presentation of the gaps was computer-generated in a semirandom but balanced fashion (i.e., each of the seven gap durations was randomly presented within each of 15 sets). Data collection began with the presentation of the eliciting stimulus and continued for $64 \mathrm{msec}$. The analog signal for each response was digitized at $1 \mathrm{kHz}$ and converted to grams using a previously determined calibration curve for each load cell.

Daily response-amplitude and latency-to-onset measurements were taken from each animal's average response curve, calculated across all trials at a given gap duration. Baseline, amplitude, latency to onset, and number of responses were measured, as previously defined (Ruppert et al., 1983). Temporal auditory acuity was defined as the level of gap that was effective in producing inhibition of the ASR (as previously defined by Kellogg et al., 1983).

One male or female pup from each of 16 litters $(n=8 / \mathrm{sex})$ was assigned to one of eight age groups for gap ontogeny testing. The rats in each age group were tested for 3 consecutive days. Independent groups of rats were thus tested on PNDs 14-16, 21-23, 28-30, $35-37,49-51,63-65,77-79$, and 98-100.

\section{Data Analysis}

Each parameter of the startle response (amplitude, latency to onset, and number of responses) was analyzed separately using univariate repeated measures general linear model analyses (SAS, 1985). Analyses were conducted using age group and sex as between-subject factors; experience, gap, and all interactions were within-subject factors. Greenhouse-Geisser corrected $F$ approximations were used when appropriate. When significant interactions were found, stepdown tests were conducted to analyze for main effects. To determine gap durations where amplitude of the ASR was significantly different from the control (no-gap) amplitude, contrast tests were conducted. Best-fit polynomial trends were also defined for each function.

\section{RESULTS}

Inhibition of ASR amplitude by gaps in background noise was dependent on both age and experience. For response amplitude, this was seen as a significant age group $\times$ experience $\times$ gap interaction $[F(84,1320)=$ $3.22, p<.0001$ ]; therefore, data were analyzed separately for each age group to determine the effects of experience and gap. There was a significant experience $x$ gap interaction for all age groups subsequent to 


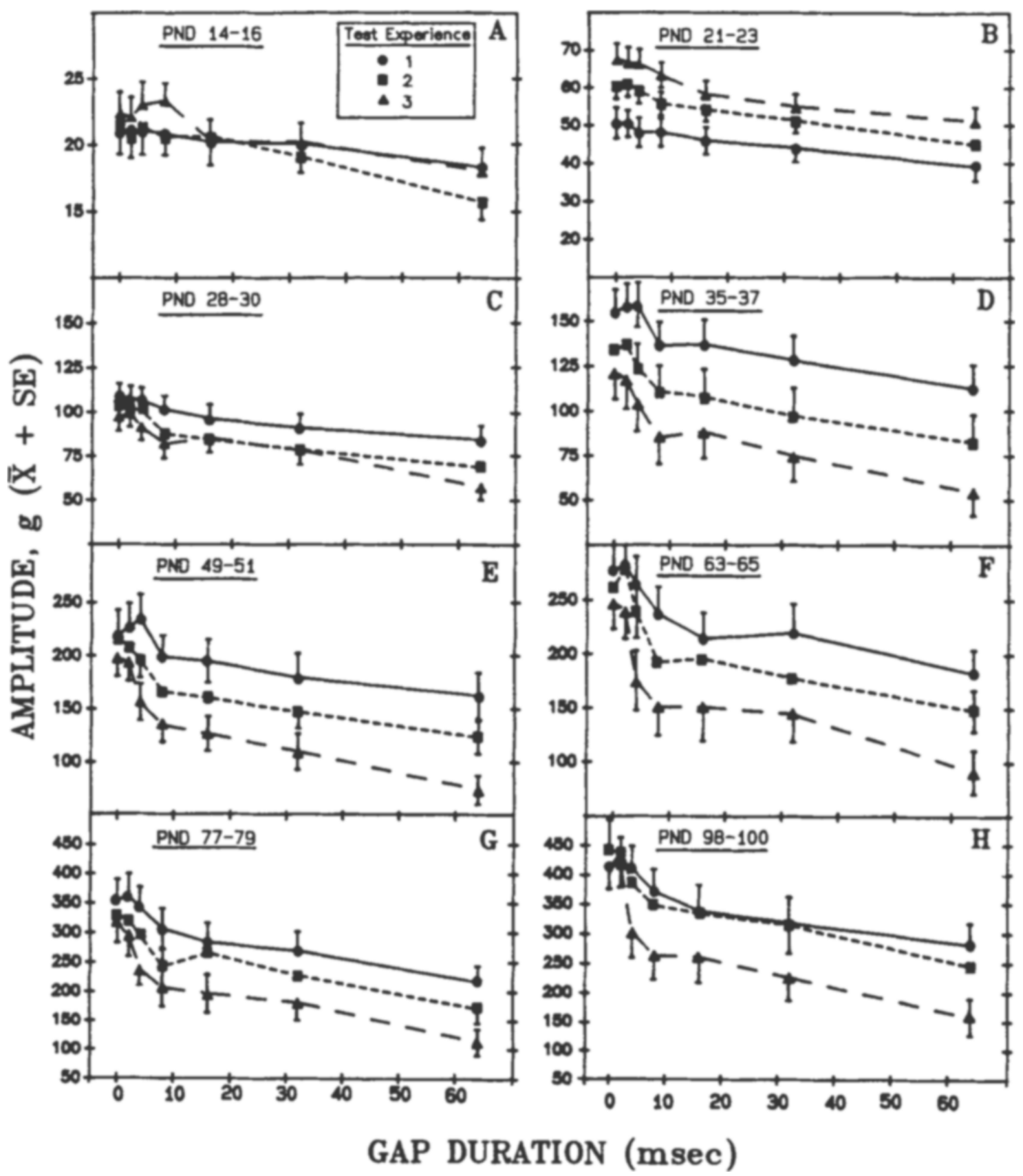

Figure 1. The effect of auditory gaps on acoustic startle response (ASR) amplitude as a function of age and experience. The data are expressed as mean $\pm S E$ ASR amplitude $(n=16$ /age group).

PND 14-16 $[F \mathrm{~s}(12,168) \geq 2.21$, all $p s \leq .05]$; therefore, the effect of gap was examined separately for each experience within those age groups.

Figure 1 shows response amplitude for each gap duration across the three daily experiences for each of the eight ages tested. On PND 14-16, response amplitude showed a significant linear decrease as gap duration increased (Figure 1A). There was a significant effect of gap, but no effect of experience or gap $\times$ experience interaction in this age group. The mean response amplitude (averaged across experience) was significantly less than that of the control (no-gap) amplitude at gaps of $16 \mathrm{msec}$ and greater.

On PNDs 21-23, response amplitude showed a significant linear decrease as a function of increasing gap duration, and a significant quadratic trend by PND 23 (Figure 1B). Gaps of 16-64 msec produced significant inhibition of amplitude on PNDs 21 and 23, and gaps of 8-64 msec reduced amplitudes on PND 22.

On PND 28, response amplitudes showed significant linear and quadratic trends; in contrast, linear and cubic trends were significant on PNDs 29 and 30 (Figure 1C). 
Gaps of 8-64 msec produced significant inhibition of the response for all experiences in this age group.

On PNDs 35-37, amplitudes showed significant linear and cubic trends for all experiences (Figure 1D). There was significant inhibition of the amplitude following gaps of $8 \mathrm{msec}$ and greater on PND 35, and gaps of $4 \mathrm{msec}$ and greater by PNDs 36 and 37 .

On PNDs 49 and 50, the response curves showed both linear and quadratic trends, and on PND 51 linear and cubic trends were significant (Figure 1E). Again, gaps of $8 \mathrm{msec}$ and greater produced significant inhibition of amplitudes on PND 49, and on PNDs 50 and 51, inhibition was seen following gaps of $4 \mathrm{msec}$ and greater.

On PNDs 63-65, amplitude showed significant linear and cubic trends for all experiences (Figure 1F). Gaps of $8 \mathrm{msec}$ and greater consistently produced inhibition of the response on PND 63, and gaps of $4 \mathrm{msec}$ and greater produced inhibition on PNDs 64 and 65. This pattern of inhibition also was seen for both the PND 77-79 and the PND 98-100 age groups (Figures $1 \mathrm{G}$ and $1 \mathrm{H}$ ).

The magnitude of inhibition was also affected by age, experience, and gap duration. These data are shown separately for each level of gap across age in Figure 2. There was no inhibition following gaps of $2 \mathrm{msec}$ at any age (Figure 2A). Gaps of $4 \mathrm{msec}$ did not produce inhibition in naive rats at any age; however, beginning on PND 36, inhibition was present for the second and third experiences (Figure 2B). At 4-msec gaps, the magnitude of inhibition increased from $0 \%$ to $15 \%$ for the second experience throughout the experiment. For the third experience, inhibition increased from $0 \%$ to $31 \%$ and began to plateau after PND 65 (Figure 2B).

Gaps of $8 \mathrm{msec}$ produced consistent inhibition of the response for all experiences by PND 28 (Figure 2C). Again, the magnitude of inhibition, for gaps of $8 \mathrm{msec}$, increased with age and experience. There was little change

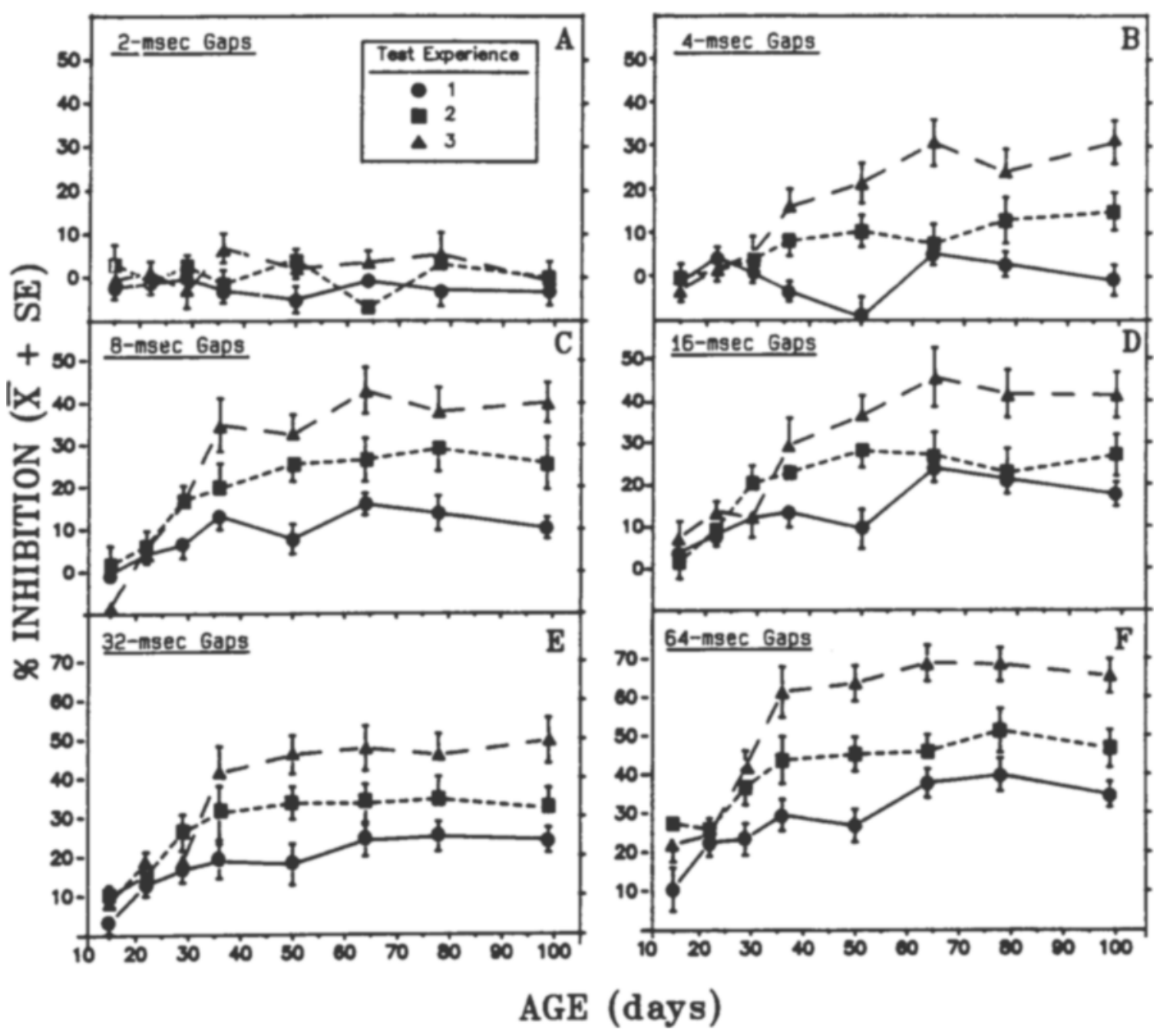

Figure 2. Ontogeny of gap inhibition. The data are redrawn from Figure 1 and are expressed as mean $\pm S E$ percent decrease from the blank-gap trials ( $n=16$ /age group). 
across age for the first experience $(0 \%-10 \%)$; however, for the second and third experiences, inhibition increased from $0 \%$ to $27 \%$ and from $0 \%$ to $40 \%$, respectively (Figure 2C). Again, the response began to asymptote after PND 65; this profile was seen for all experiences.

For 16-msec gaps, inhibition increased from $4 \%$ to $18 \%$ for the first experience, from $4 \%$ to $28 \%$ for the second experience, and from $4 \%$ to $42 \%$ for the third experience (Figure 2D). As with 8-msec gaps, an asymptotic level of inhibition was apparent by PND 65 for all experiences.

For gaps of $32 \mathrm{msec}$, inhibition increased with age from $3 \%$ to $25 \%$ for the first experience, from $8 \%$ to $33 \%$ for the second experience, and from $10 \%$ to $50 \%$ for the third experience (Figure 2E). Gaps of $64 \mathrm{msec}$ also produced increased inhibition as a function of age and experience: inhibition ranged from $10 \%$ to $35 \%$ for the first experience, from $22 \%$ to $47 \%$ for the second experience, and from $27 \%$ to $65 \%$ for the third experience (Figure 2F). The profile of gap inhibition for gaps of 32 and $64 \mathrm{msec}$ followed the previous pattern of leveling off on PND 65 for the first experience; however, for the second and third experiences, inhibition began to level off much earlier, on PND 35 (Figure 2F).

The lowest level of gap that produced significant inhibition of the ASR is shown as a function of age and experience in Figure 3. Auditory acuity in the naive rat first occurred at gaps of $16 \mathrm{msec}$ and decreased to $8 \mathrm{msec}$ by PND 28. Repeated testing did not alter the level of acuity prior to PND 35; however, after that time the level of acuity decreased from 8 to $4 \mathrm{msec}$ from the first to the second or third test session (Figure 3). There was no change in the level of acuity from the second to the third experience at any age.
For latency to onset, there was a significant age group $\times$ experience $\times$ gap interaction $[F(84,1320)=1.53$, $p<.01]$. However, when separate age groups were examined, consistent effects were not found either for gap or for experience. The interaction was the result of significant experience $\times$ gap interactions from Age Groups 35-100. There was no replicable effect of experience across age groups at any particular gap (e.g., at some ages there was a decrease in latency across the 3 test days, whereas at different ages, latency increased or remained the same across the 3 days of testing). There were no significant effects on the number of responses. In all cases, the number of responses was greater than $95 \%$. Data are not shown for onset latency or for the number of responses.

\section{DISCUSSION}

All measures of gap inhibition were affected by age, gap duration, and experience. Although gap inhibition was present at all ages, both the minimum gap duration, which produced significant inhibition, and the magnitude of inhibition changed as a function of age and experience. Between PND 14 and PND 28, the level of gap detection (acuity) decreased from 16 to $8 \mathrm{msec}$. This gradual improvement in acuity as a function of age is consistent with the ontogenetic pattern of a similar measure of auditory temporal acuity in children (Davis \& McCroskey, 1980). No further improvement in acuity was seen (through PND 98) in the naive rat. Therefore, the mature level of acuity found in this study for naive rats $(8 \mathrm{msec})$ was obtained by PND 28. The magnitude of inhibition produced by gaps was also affected by age. In naive rats, the on-

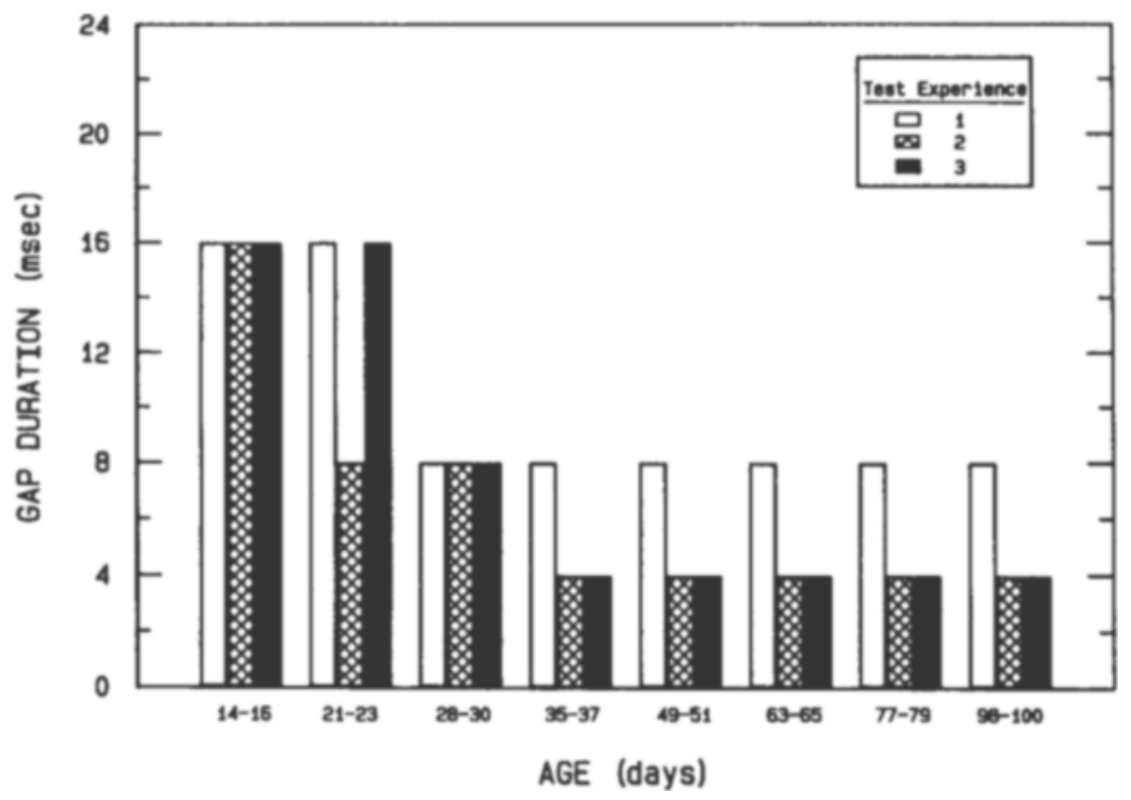

Figure 3. Ontogeny of gap acuity. The data indicate the shortest gap duration at which amplitude was significantly decreased from the blank-gap amplitude, using Tukey's a post hoc comparisons of means $(n=16$ /age group). 
togenetic profile of inhibition showed a gradual increase with age, with maximal inhibition (at 64-msec gaps) ranging from $10 \%$ to $35 \%$.

The effect of experience on gap inhibition was dependent on the age of the rats. In the youngest age group (PND 14-16), experience was not a significant factor. The response was inhibited following gaps of $16 \mathrm{msec}$ and greater, regardless of experience. The effect of experience became more pronounced in older rats. Prior to PND 35, experiential effects were seen primarily as increased inhibition at the longer gap durations (32 and $64 \mathrm{msec}$ ) without affecting gap acuity. However, beginning on PND 35, both the magnitude of inhibition and acuity were affected by experience. With the addition of a second test experience on PND 36, acuity improved to $4 \mathrm{msec}$. This improvement in acuity was seen for the second experience in all subsequent age groups. In fact, further improvement in acuity was not seen at any age or experience. Therefore, the adult pattern of gap acuity over the course of three test experiences was established by PND 35-37. The effect of test experience was more pronounced for the magnitude of gap inhibition than for acuity. Maximal inhibition (at 64-msec gaps) increased dramatically with experience. In contrast to the naive rat, maximal inhibition for the third test experience ranged from $20 \%$ to $67 \%$ over the course of the experiment. Beginning on PND 35, each test experience produced an additional $20 \%$ increase in inhibition, for gaps of $4 \mathrm{msec}$ or greater. Therefore, although the addition of a third test experience did not alter acuity, the magnitude of inhibition did continue to increase. Experience has been acknowledged to be an important factor in the testing of auditory temporal acuity of humans: it is routine to allow human subjects a brief "practice" session prior to the final determination of acuity thresholds (Davis \& McCroskey, 1980; Fitzgibbons, 1983).

The results of this study do not entirely agree with the previous report of gap ontogeny (Kellogg et al., 1983). In the present study, inhibition was seen on PND 14, much earlier than the previously reported age of "slight inhibition" on PND 25. The presence of gap inhibition at this early age is in agreement with the earliest appearance of both sensitization and prepulse inhibition, but it is significant that it occurred only at the longer gaps. The ontogenetic profile of acuity showed a gradual improvement, from 16 to $8 \mathrm{msec}$, between PND 14 and PND 28 . In contrast, the previous report of gap ontogeny did not find inhibition at any level of gap $(0,3,4,5,6,7,12$, or $24 \mathrm{msec}$ ) until the adult level was present, on PND 28. Both the present study and the one by Kellogg et al. found the mature level of acuity on PND 28; however, the major discrepancy between the two findings is the level of acuity ( 3 vs. $8 \mathrm{msec}$ ). In the present study, acuity at a comparable level $(4 \mathrm{msec})$ was not seen until PND 36 . In addition, this level of acuity was achieved only in experienced rats, not in naive animals. Acuity at $4 \mathrm{msec}$ may be possible at an earlier age; however, the results of the present study indicate that additional test experiences would be required. It is unlikely that any further improvement in acuity (less than $4 \mathrm{msec}$ ) could have been demonstrated, since the threshold of ASR gap inhibition in adult rats has been defined at $3.5 \mathrm{msec}$ (Ison, 1982). This level of detection is also similar to that found in human subjects (Ison \& Pinckney, 1983).

The discrepancies between the present report and the previous report of gap ontogeny (Kellogg et al., 1983) may be due to differences in the stimulus parameters used. The present study was specifically designed to define the ontogeny of gap inhibition; therefore, parameters were selected to maximize the occurrence of gap inhibition. Since inhibition shows a graded response as a function of gap duration (Ison, 1982; Ison \& Pinckney, 1983), gaps of very long durations were selected in our study. We also employed a higher background noise level than was used by Kellogg et al. ( 80 vs. $70 \mathrm{~dB}$ ). Frequency, bandwidth, and intensity of background noise have been shown to affect auditory acuity in humans (Fitzgibbons, 1983; Shailer \& Moore, 1983).

The results of the present study demonstrate that inhibition of the ASR in response to gaps in background noise is present on PND 14, the earliest age tested. In addition, our results indicate that testing for three daily sessions alters the magnitude of inhibition at all ages and improves the acuity of rats older than PND 35. However, there are two issues regarding repeated testing and its role on gap inhibition that require further investigation. First, since gap inhibition was present on Day 1 of testing (PND 14), the earliest age at which gap inhibition is present may not have been determined. Further work is necessary to determine whether gap detection develops concurrently with the ASR, or at the same age that reflex modification using sensitization and prepulse develops. Second, experiential effects described in this paper are limited to three daily test sessions. Since the magnitude of inhibition continued to increase across the first three sessions, further work is needed to characterize the effect of additional test experiences on the magnitude of inhibition.

\section{REFERENCES}

AVERY, D. L., \& SPYKer, J. M. (1977). Foot tattoo of neonatal mice. Laboratory Animal Science, 27, 110-112.

Crofton, K. M., Sheets, L. P., Dean, K. F., \& Peele, D. B. (1988). The effects of experience on gap inhibition of the acoustic startle response (ASR). Society for Neuroscience Abstracts, 4, 60.

Crowley, D. E., \& Hepp-Raymond, M. C. (1966). Development of cochlear function in the ear of the infant rat. Journal of Comparative \& Physiological Psychology, 2, 427-432.

DAvis, M. (1984). The mammalian startle response. Neural Mechanisms of Startle Behavior, 10, 287-335.

Davis, S. M., \& MCCroskey, R. L. (1980). Auditory fusion in children. Child Development, 51, 75-80.

Dean, K. F., Sheets, L. P., Crofton, K. M., Reiter, L. W. (1988). The effect of age and experience on inhibition of the acoustic startle response (ASR) by gaps in background noise. Society for Neuroscience Abstracts, 4, 425.

FitzGibrons, P. J. (1983). Temporal gap detection in noise as a func- 
tion of frequency, bandwidth and level. Journal of the Acoustical Society of America, 74, 67-72.

Hoffman, H. S., \& Fleshler, M. (1963). Startle reaction: Modification by background acoustic stimulation. Science, 141, 928-930.

Hoffman, H. S., Ison, J. R. (1980). Reflex modification in the domain of the startle: I. Some empirical findings and their implications for how the nervous system processes sensory input. Psychological Review, 87, 175-189.

Hoffman, H. S., \& SEarle, J. L. (1965). Acoustic and temporal factors in the evocation of startle. Journal of Comparative \& Physiological Psychology, 60, 53-58.

IsoN, J. R. (1982). Temporal acuity in auditory function in the rat: Reflex inhibition by brief gaps in noise. Joumal of Comparative \& Physiological Psychology, 96, 945-954.

Ison, J. R. (1988, December). Behavioral methods applicable to laboratory animals and humans. Paper presented at the Third International Symposium on Neurobehavioral Methods in Occupational and Environmental Health, Washington, DC.

Ison, J. R., \& PINCKney, L. A. (1983). Reflex inhibition in humans: Sensitivity to brief silent periods in white noise. Perception \& Psychophysics, 34, 84-88.

Kellogg, C., Ison, J. R., \& Miller, R. K. (1983). Prenatal diazepam exposure: Effects on auditory temporal resolution in rats. $P s y$ chopharmacology, 79, 332-337.

Kellogg, C., Tervo, D., Ison, J., \& Parisi, T. (1980). Prenatal exposure to diazepam alters behavioral development in rats. Science, 207, 205-207.
Parisi, T. P., \& Ison, J. R. (1979). Development of the acoustic startle response in the rat: Ontogenetic changes in the magnitude of inhibition by prepulse stimulation. Developmental Psychobiology. 12, 219-230.

PARISI, T. P., \& IsON, J. R. (1981). Ontogeny of control over the acoustic startle reflex by visual prestimulation in the rat. Developmental Psychobiology, 14, 311-316.

Ruppert, P. H., Dean, K. F., \& Reiter, L. W. (1983). Development and behavioral toxicity following acute postnatal exposure of rat pups to trimethyltin. Neurobehavioral Toxicology \& Teratology, 5, 421-429.

SAS Institute, INC. (1985). SAS user's guide: Statistics (Version 5). Cary, NC: Author.

Shaller, M. J., Moore, B. C. J. (1983). Gap detection as a function of frequency, bandwidth and level. Journal of the Acoustical Society of America, 74, 467-473.

Sheets, L. P., Dean, K. F., \& Reiter, L. W. (1988). Ontogeny of the acoustic startle response and sensitization to background noise in the rat. Behavioral Neuroscience, 102, 706-713.

Young, L. S., \& Fechter, L. D. (1983). Reflex inhibition procedures for animal audiometry: A technique for assessing ototoxicity. Journal of the Acoustical Society of America, 73, 1686-1693.

(Manuscript received June 8, 1989;

revision accepted for publication January 9, 1990.)

\section{Notices and Announcements}

\section{Fourth Conference on the Neurobiology of Learning and Memory University of California, Irvine October 17-20, 1990}

The Fourth Conference on the Neurobiology of Learning and Memory will be held at the Center for the Neurobiology of Learning and Memory at the University of California, Irvine, October 17-20, 1990.

The focus of the conference will be on Memory: Organization and Locus of Change-distribution of learning-induced brain activity, functional roles of brain systems, and locus of cellular change. A poster session will be included in the program.

Registration will be limited. Complete program and application information may be obtained by contacting James L. McGaugh, 1990 Conference Information, Center for the Neurobiology of Learning and Memory, University of California, Irvine, CA 92717-Phone, (714) 856-5193 or 5401; FAX, (714) 856-8481. 